\title{
COMMENTARY
}

\section{Defining the boundaries of bedside pulse contour analysis: dynamic arterial elastance}

\author{
Michael R Pinsky \\ See related research by García et al., http://ccforum.com/content/15/1/R15
}

\begin{abstract}
Assessment of vasomotor tone is essential in defining appropriate resuscitation strategies for the hypotensive patient. Although changes in mean arterial pressure to cardiac output define arterial resistance, resistance is only one component of vasomotor tone. Compliance is the other component. The reciprocal of compliance is arterial elastance (Ea). Importantly, dynamic Ea can be estimated by the pulse pressure variation to stroke volume variation relation. Dynamic Ea is only one component of vasomotor tone, however, and increases in pulse pressure may not be proportional to increases in mean arterial pressure. Also, devices that use the arterial pressure pulse to calculate the stroke volume have an inherent bias that is different amongst devices based on their transfer function algorithms. The use of dynamic Ea for clinical decision-making thus needs to be validated separately for different devices and types of patients.
\end{abstract}

Resuscitation from hypotensive circulatory shock is often more complex than just giving intravascular fluids. Even in fluid-responsive hypotensive patients, vital organ perfusion may remain compromised despite fluid infusion if the mean arterial pressure (MAP) does not also increase with increasing cardiac output (CO). García and colleagues suggested an approach that would predict whether the hypotensive patient would increase their MAP in response to intravascular fluid loading [1].

There is a tight correlation between positive-pressure ventilation-induced changes in arterial pulse pressure (PP), called pulse pressure variation (PPV), and fluid responsiveness [2]. PPV is calculated as the ratio of difference between maximum and minimum PP to their

Correspondence: pinskymr@upmc.edu

Department of Critical Care Medicine, University of Pittsburgh, 606 Scaife Hall, 3550 Terrace Street, Pittsburgh, PA 15261, USA mean as assessed over about 5 breaths or 20 seconds. The greater the PPV, the greater the $\mathrm{CO}$ increase. Not surprisingly, subsequent studies showed that similarly calculated stroke volume variation (SVV), when measured independently, also predicted volume responsiveness [3]. Importantly, PP is created by stroke volume (SV) into the central arterial compartment as quantified by a transfer function [4]. If arterial elastance (Ea) and compliance remain constant, then aortic PP will vary directly with $\mathrm{SV}$. If the bedside physician wants to know whether their hypotensive patient will increase their MAP in response to fluid loading, then they need to know two things. First, is the patient volume responsive? If the patient is not responsive, then volume loading will not increase $\mathrm{CO}$. Second, the physician needs to know the patient's vasomotor tone. If the patient has marked vasodilation, as commonly occurs in septic shock, then MAP may not increase in response to fluid loading even if $\mathrm{CO}$ does.

To know whether a fluid-responsive patient is also pressure responsive, one needs to assess vasomotor tone. The relation between dynamic changes in MAP to CO defines arterial resistance. But resistance is only one aspect of arterial tone. Vasomotor tone is defined by both the resistance and compliance. The reciprocal of compliance is Ea, which defines the $\mathrm{PP} / \mathrm{SV}$ relation [5]. Increasing vasomotor tone increases both MAP and PP relative to $\mathrm{CO}$ and $\mathrm{SV}$. If one knew both PPV and SVV, their ratio would define a dynamic $\mathrm{Ea}\left(\mathrm{Ea}_{\mathrm{dyn}}\right)$ [6]. Theoretically, MAP and PP should co-vary with changes in $\mathrm{CO}$ if the heart rate remains constant. We previously predicted that $\mathrm{PPV} / \mathrm{SVV}>0.8$ would define pressureresponsive subjects if $\mathrm{CO}$ increased [7].

Relative to this construct, García and colleagues examined the ability of the PPV/SVV ratio, deferred to as $\mathrm{Ea}_{\text {dyn' }}$, to predict changes in MAP in 25 hypotensive patients with preserved volume responsiveness (defined as MAP $<65 \mathrm{mmHg}$ or systolic blood pressure $<90 \mathrm{mmHg}$ and SVV >10\%) [1]. Using a standard $500 \mathrm{ml}$ colloid fluid bolus, the authors defined MAP responders as those with a $>15 \%$ increase in MAP. They found that a baseline $\mathrm{Ea}_{\mathrm{dyn}}$ value $>0.89$ predicted $>15 \%$ MAP increase after fluid administration with a sensitivity of $94 \%$ (95\% confidence 
interval $=69.8$ to $99.8 \%)$ and a specificity of $100 \%(95 \%$ confidence interval $=66.4$ to $100 \%)$. Their clinically derived $\mathrm{Ea}_{\text {dyn }}$ threshold of $>0.89$ is remarkable similar to our $>0.8$ value based on vascular modeling. Importantly, as long as the PPV and SVV values are great enough to define a slope, this relationship will remain constant and predictive even during spontaneous ventilation and with cardiac arrhythmias because $\mathrm{Ea}_{\text {dyn }}$ is independent of volume responsiveness. Since all of the commercially available arterial pressure-derived $\mathrm{CO}$ monitoring devices report PPV and SVV, this added $\mathrm{Ea}_{\mathrm{dyn}}$ parameter to define those patients needing vasopressors earlier in their management should have a significant impact on resuscitation efficacy.

Before embracing this approach and these findings totally, caution needs to be used in its routine bedside application. Importantly, $\mathrm{Ea}_{\mathrm{dyn}}$ is not a direct measure of $\mathrm{Ea} . \mathrm{Ea}_{\mathrm{dyn}}$ is a measure of arterial stiffness, which is itself partially determined by vasomotor tone. Baseline Ea is not constant. Ea is lower in premenopausal women than in age-matched men. Ea increases with age, with the expression of atherosclerosis and with aortic crossclamping during aortic vascular procedures. For all these conditions, however, if SV increases then PP will also increase. Regrettably, MAP may not increase as much as PP because diastolic pressure may remain constant. Furthermore, PP can be independently increased by increasing left ventricular ejection velocity [6]. To the extent that inotropic agents are being used, one may presume an increase in MAP not realized by increasing $\mathrm{CO}$ even if $\mathrm{Ea}_{\text {dyn }}>0.89$.

If PPV and SVV are independently measured, then the clinical utility becomes manifest. The above study, however, used an arterial pulse contour estimating method to derive CO and SVV [6], and herein rests a potential problem. Much attention has focused on using arterial pulse contour analysis devices at the bedside to measure $\mathrm{CO}$ and SVV from the arterial pulse. Unfortunately, most indwelling arterial catheters sample a more peripheral arterial pressure signal, whose waveform may be altered in unexpected ways as the arterial tone, pulse wave velocity and left ventricular contractility vary. Although this variability in $\mathrm{CO}$ estimates can be readily improved by calibration, it will not improve the accuracy of the $\mathrm{PPV} / \mathrm{SVV}$ relation (that is, $\mathrm{Ea}_{\mathrm{dyn}}$ ). The $\mathrm{Ea}_{\mathrm{dyn}}$ accuracy is intrinsic to the assumptions used by each device to estimate SVV. This is because PPV can be measured directly and is accurate when compared with PPV measured manually [8].

All arterial pressure-sensing devices that estimate $\mathrm{CO}$ do so by assuming a constant Ea. How, then, is it possible for an algorithm that uses arterial pressure to calculate $\mathrm{CO}$ to show differing changes in PP relative to SV over time? This is the Achilles' heel of all these pulse contour monitoring devices. The three major commercially available monitoring devices, however, do not share the same shoe size - the variance amongst these devices to estimate changing $\mathrm{CO}$ is significant [9].

García and colleagues used the FloTrac ${ }^{\bullet}$ device to make their $\mathrm{Ea}_{\mathrm{dyn}}$ measures. The FloTrac ${ }^{\circledR}$ device estimates SVV from the standard deviation of the individual arterial pressure values over single beats averaged over 20 seconds. Assuming the SV variance has a normal distribution, this assumption is valid for calculating SVV. Importantly, SVV as a time-series function may not be normally distributed during atrial fibrillation and with vigorous spontaneous inspiratory efforts. How much error such non-normal distribution would introduce into the SVV calculation is not known, but on theoretical modeling the degree of non-normal distribution would need to be great for it to affect SVV by the standard deviation method.

The other two devices are the PiCCO and the LiDCO. The PiCCO uses a proprietary algorithm based on the ventriculo-arterial coupling transfer function [3] to calculate $\mathrm{CO}$. This $\mathrm{CO}$ estimate is averaged over 20 to 30 seconds and is quite accurate [9]. However, since the $\mathrm{PiCCO}$ no longer reports individual SV values on a beatto-beat basis, it is unclear how it derives SVV. The LiDCO uses a simple power transfer function to estimate $\mathrm{SV}$ on a beat-to-beat basis and calculates $\mathrm{CO}$ from the mean SV values. To the extent that this power transfer function is accurate over time, the SVV estimates should also be accurate. Since this device does not use the pulse contour, the LiDCO remains accurate with dampened arterial pressure signals.

Accordingly, although each device reports PPV and SVV values, the cross-correlation amongst devices based on their different algorithms is poor [9]. Validation of $\mathrm{Ea}_{\mathrm{dyn}}$ thus needs to be done independently both for each device and for different types of patients before this new bedside parameter is used for clinical decision-making.

\section{Abbreviations}

CO, cardiac output; Ea, arterial elastance; Ea ${ }_{\text {dyn }}$ dynamic arterial elastance; MAP, mean arterial pressure; PP, pulse pressure; PPV, pulse pressure variation; SV, stroke volume; SW, stroke volume variation.

\section{Conflicts of interest}

MRP is a consultant for Edwards LifeSciences and LiDCO Ltd.

\section{Acknowledgements}

The present work was supported in part by National Institutes of Health grant HL67181.

Published: 9 February 2011

\section{References}

1. García MIM, Cano AG, Romero MG: Dynamic arterial elastance to predict arterial pressure response to volume loading in preload-dependent patients. Crit Care 2011, 15:R15.

2. Michard F, Chemla D, Richard C, Wysocki M, Pinsky MR, Lecarpentier Y, Teboul $J \mathrm{~L}$ : Clinical use of respiratory changes in arterial pulse pressure to monitor 
the hemodynamic effects of PEEP. Am J Respir Crit Care Med 1999, 159:935-939.

3. Monnet X, Rienzo M, Osman D, Anguel N, Richard C, Pinsky MR, Teboul JL: Esophageal Doppler monitoring predicts fluid responsiveness in critically ill ventilated patients. Intensive Care Med 2005, 31:1195-1201.

4. Hamilton WF, Remington $W$ : The measurement of the stroke volume from the pressure pulse. Am J Physiol 1947, 148:14-24

5. Kelly RP, Ting CT, Yang TM, Liu CP, Maughan WL, Chang MS, Kass DA: Effective arterial elastance as index of arterial vascular load in humans. Circulation 1992, 86:513-521.

6. Chemla D, Hebert UL, Coirault C, Zamani K, Suard I, Colin P, Lecarpentier Y: Total arterial compliance estimated by stroke volume-to-aortic pulse pressure ratio in humans. Am J Physiol 1998, 274: H500-H505.

7. Pinsky MR: Protocolized cardiovascular management based on ventricular-arterial coupling. In Functional Hemodynamic Monitoring. Edited by Pinsky MR, Payen D. Berlin: Springer-Verlag; 2006:381-395.

8. Hadian M, Severyn D, Pinsky MR: The effects of vasoactive drugs on pulse pressure and stroke volume variation in post-operative ventilated patients. J Crit Care 2010. doi:10/10-1016/j.jcrc.2010.08.018.

9. Hadian M, Kim H, Severyn DA, Pinsky MR: Cross-comparison of cardiac output trending accuracy of LiDCO, PiCCO, FloTrac and pulmonary artery catheters. Crit Care 2010, 14:R212.

doi:10.1186/cc9986

Cite this article as: Pinsky MR: Defining the boundaries of bedside pulse contour analysis: dynamic arterial elastance. Critical Care 2011, 15:120. 